\title{
Extending the random-phase approximation for electronic correlation energies: the renormalized adiabatic local density approximation
}

\author{
Olsen, Thomas; Thygesen, Kristian S.
}

Published in:

Physical Review B Condensed Matter

Link to article, DOI:

10.1103/PhysRevB.86.081103

Publication date:

2012

Document Version

Publisher's PDF, also known as Version of record

Link back to DTU Orbit

Citation (APA):

Olsen, T., \& Thygesen, K. S. (2012). Extending the random-phase approximation for electronic correlation energies: the renormalized adiabatic local density approximation. Physical Review B Condensed Matter, 86(8), 081103. https://doi.org/10.1103/PhysRevB.86.081103

\section{General rights}

Copyright and moral rights for the publications made accessible in the public portal are retained by the authors and/or other copyright owners and it is a condition of accessing publications that users recognise and abide by the legal requirements associated with these rights.

- Users may download and print one copy of any publication from the public portal for the purpose of private study or research.

- You may not further distribute the material or use it for any profit-making activity or commercial gain

- You may freely distribute the URL identifying the publication in the public portal 


\title{
Extending the random-phase approximation for electronic correlation energies: The renormalized adiabatic local density approximation
}

\author{
Thomas Olsen* and Kristian S. Thygesen \\ Center for Atomic-Scale Materials Design, Department of Physics, Technical University of Denmark, DK-2800 Kongens Lyngby, Denmark
}

(Received 3 May 2012; published 15 August 2012)

\begin{abstract}
The adiabatic connection fluctuation-dissipation theorem with the random phase approximation (RPA) has recently been applied with success to obtain correlation energies of a variety of chemical and solid state systems. The main merit of this approach is the improved description of dispersive forces while chemical bond strengths and absolute correlation energies are systematically underestimated. In this work we extend the RPA by including a parameter-free renormalized version of the adiabatic local-density (ALDA) exchange-correlation kernel. The renormalization consists of a (local) truncation of the ALDA kernel for wave vectors $q>2 k_{F}$, which is found to yield excellent results for the homogeneous electron gas. In addition, the kernel significantly improves both the absolute correlation energies and atomization energies of small molecules over RPA and ALDA. The renormalization can be straightforwardly applied to other adiabatic local kernels.
\end{abstract}

DOI: 10.1103/PhysRevB.86.081103

PACS number(s): 71.15.Mb, 31.15.E-

Increasing computational resources has recently boosted a major interest in calculating electronic correlation energies from first principles using the adiabatic connection fluctuationdissipation theorem (ACDF). ${ }^{1-4}$ The computational cost for such methods is much higher than traditional correlation functionals in density functional theory, but has the great advantage that it includes nonlocal effects and does not rely on error cancellation between exchange and correlation. The random phase approximation (RPA) represents the simplest approach to ACDF calculations and has already been applied to a broad range of electronic structure problems. ${ }^{5-11}$ While the nonlocality of RPA makes it superior to semilocal functionals when dispersive interactions are important, ${ }^{5,7,10,12-14}$ the accuracy for molecular atomization energies is comparable to that of the Perdew-Burke-Ernzerhof (PBE) functional., ${ }^{3,6}$ The relatively poor performance for atomization energies can be attributed to a deficient description of short-range correlation effects. Furthermore, total correlation energies are severely underestimated in RPA and an accurate description of energy differences is highly dependent on detailed error cancellation. A simple and intuitively appealing idea to remedy this problem was proposed by Yan et al. ${ }^{15}$ (RPA+), however, the method does not seem to improve atomization energies although total correlation energies are much better described. ${ }^{3,6,7}$ From a perturbative point of view, RPA can be improved by including a screened second-order exchange term (SOSEX), ${ }^{16}$ which exactly cancels the one-electron self-correlation energy of RPA, albeit with a significant increase in computational cost. In addition, it has been shown that RPA results can be improved by explicitly including single excitation terms, which correct the use of non-self-consistent input orbitals. ${ }^{17}$

From the point of view of time-dependent density functional theory (TDDFT), it is natural to try to improve the description of short-range correlation effects by extending RPA with an exchange-correlation kernel. For the homogeneous electron gas (HEG), this approach has been analyzed for a range of known exchange-correlation kernels ${ }^{1,18}$ and led to the construction of new adiabatic kernels fitted to reproduce the HEG correlation energy. ${ }^{4,19}$ So far, it seems that for accurate total energy calculations, the nonlocality of exchange-correlation kernels is very important, whereas the frequency dependence is less critical. Moreover, the pair-distribution function derived from any local approximation for the exchange-correlation kernel exhibits an unphysical divergence at the origin. ${ }^{2}$ While correlation energies are still well defined, the divergence makes it very hard to converge correlation energies based on local kernels. Recently, a frequency-dependent exact exchange kernel has been shown to produce accurate correlation energies for atoms and molecules. ${ }^{20,21}$ However, the computational cost of this approach is significantly larger than that of RPA and the method may not be directly applicable to periodic systems.

In this Rapid Communication we derive a nonlocal exchange-correlation kernel which does not contain any fitted parameters. The construction is based on a renormalization of the HEG correlation hole, which removes the divergence of the pair-distribution function and brings total correlation energies much closer to experimental values than both RPA and local approximations for the kernel.

From the adiabatic connection and fluctuation-dissipation theorem, it follows that the correlation energy of an electronic system can be written

$$
E_{c}[n]=-\int_{0}^{1} d \lambda \int_{0}^{\infty} \frac{d \omega}{2 \pi} \operatorname{Tr}\left[v \chi^{\lambda}(i \omega)-v \chi^{\mathrm{KS}}(i \omega)\right] .
$$

Here $\chi^{\mathrm{KS}}$ is the exact Kohn-Sham response function and $\chi^{\lambda}$ is the interacting response function of a system where the electron-electron interaction $v$ has been replaced by $\lambda v$. Using TDDFT, one may express the interacting response function in terms of the Kohn-Sham response function as

$$
\chi^{\lambda}=\chi^{\mathrm{KS}}+\chi^{\mathrm{KS}} f_{\mathrm{Hxc}}^{\lambda} \chi^{\lambda},
$$

where $f_{\mathrm{Hxc}}^{\lambda}=\lambda v+f_{\mathrm{xc}}^{\lambda}$ is the Hartree-exchange-correlation kernel at coupling strength $\lambda$. The simplest approximation for $f_{\mathrm{Hxc}}^{\lambda}$ is the random phase approximation where the exchange-correlation part is neglected. A natural next step is to include an approximation for the exchange-correlation kernel. In particular, one could try the adiabatic local density approximation (ALDA) kernel

$$
f_{\mathrm{xc}}^{\mathrm{ALDA}}[n]\left(\mathbf{r}, \mathbf{r}^{\prime}\right)=\delta\left(\mathbf{r}-\mathbf{r}^{\prime}\right) f_{\mathrm{xc}}^{\mathrm{ALDA}}[n],
$$



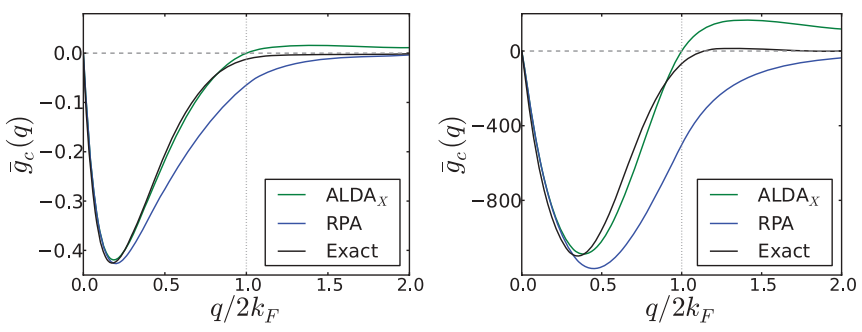

FIG. 1. (Color online) Fourier transform of the coupling constant averaged correlation hole for the homogeneous electrons gas. Left: $r_{s}=1$. Right: $r_{s}=10$.

where $f_{\mathrm{xc}}^{\mathrm{ALDA}}[n]=\left.\frac{d^{2}}{d n^{2}}\left(n e_{\mathrm{xc}}^{\mathrm{HEG}}\right)\right|_{n=n(\mathbf{r})}$. In the following we will only consider the exchange part of the adiabatic kernel, since it has the simplifying property that $f_{x}^{\lambda}=\lambda f_{x}$. Additionally, we expect the effect of including a kernel in Eq. (2) will be dominated by the exchange contributions. As it turns out, the kernel Eq. (3) does not improve on total correlation energies ${ }^{1}$ or molecular atomization energies ${ }^{2}$ and is plagued by convergence problems related to the divergence of the pair-distribution function.

For the homogeneous electron gas the problem is naturally analyzed in reciprocal space where an accurate parametrization of the correlation hole is known. ${ }^{1,22}$ In Fig. 1 we show the exact coupling constant averaged correlation hole of the homogeneous electron gas and compare with RPA and ALDA $_{X}$ results. Whereas RPA underestimates the value at a large range of $q$ values, $\operatorname{ALDA}_{X}$ gives a reasonable description at small $q$ but overestimates the value for $q>2 k_{F}$. The $\operatorname{ALDA}_{X}$ correlation hole becomes zero when $f_{\mathrm{Hx}}^{\lambda}=0$, which happens exactly at $q=2 k_{F}$. The divergence of the pair-distribution function originates from the slowly decaying tail at large $q$ where $f_{\mathrm{Hxc}}$ is complete dominated by the $q$-independent

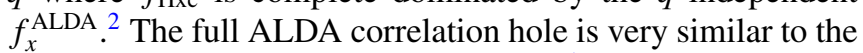
$\mathrm{ALDA}_{X}$ correlation hole displayed here. ${ }^{1}$

The correlation energy is essentially given by the integral of the coupling constant averaged correlation hole. Despite the divergent pair-distribution function, the $\mathrm{ALDA}_{X}$ correlation energy is well defined but converges very slowly due to the slow decay of $\bar{g}(q)$ at large $q$. From the shape of the correlation hole it is expected that RPA underestimates the correlation energy, while $\mathrm{ALDA}_{X}$ overestimates it. Since the bad behavior of $\operatorname{ALDA}_{X}$ primarily comes from large values of $q$, it is now tempting to introduce a renormalized $\operatorname{ALDA}_{X}$ correlation energy obtained by cutting the $q$ integral at the zero point of $\bar{g}(q)$. The result is shown in Fig. 2 along with RPA, Petersilka-Gossmann-Gross (PGG), ${ }^{23}$ and ALDA ${ }_{X}$ correlation energies. It is seen that the renormalized $\operatorname{ALDA}_{X}$ gives a remarkable improvement compared to RPA, $\operatorname{ALDA}_{X}$, and PGG. Except for the $r_{s} \rightarrow 0$ limit, it also performs better than the functionals proposed by Corradini et al. ${ }^{24}$ and the static version of the Richardson-Ashcroft kernel ${ }^{25}$ (not shown), which were fitted to quantum Monte Carlo data and derived from many-body perturbation theory, respectively. ${ }^{1}$

For the homogeneous electron gas, the cutoff is equivalent to using the Hartree-exchange-correlation kernel

$$
f_{\mathrm{Hxc}}^{\mathrm{rALDA}}[n](q)=\theta\left(2 k_{F}-q\right) f_{\mathrm{Hx}}^{\mathrm{ALDA}}[n] .
$$

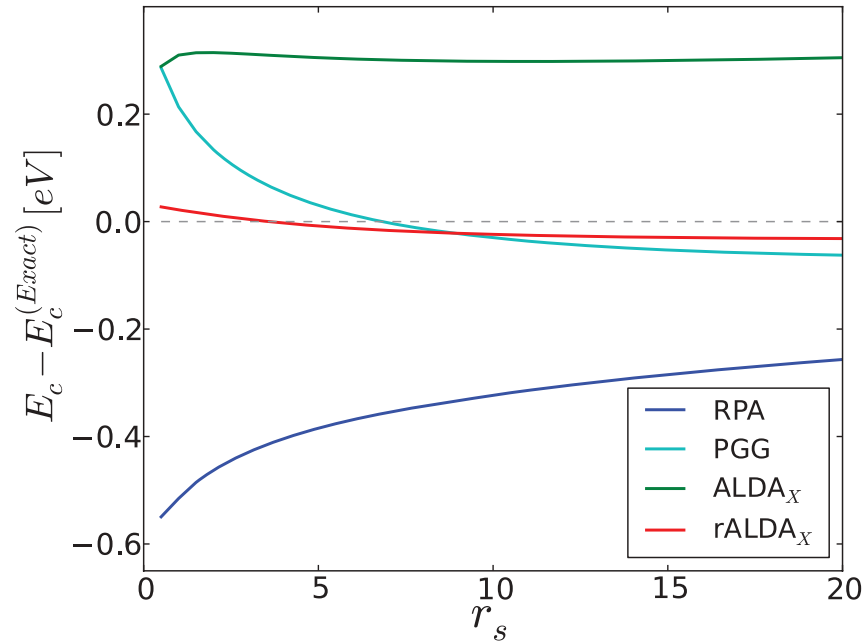

FIG. 2. (Color online) Correlation energy per electron of the homogeneous electron gas evaluated with different approximations for $f_{\mathrm{xc}}$.

Fourier transforming this expression yields

$$
\begin{aligned}
f_{\mathrm{Hxc}}^{\mathrm{rALDA}}[n](r) & =f_{x}^{\mathrm{rALDA}}[n](r)+v^{r}[n](r), \\
f_{x}^{\mathrm{rALDA}}[n](r) & =\frac{f_{x}^{\mathrm{ALDA}}[n]}{2 \pi^{2} r^{3}}\left[\sin \left(2 k_{F} r\right)-2 k_{F} r \cos \left(2 k_{F} r\right)\right], \\
v^{r}[n](r) & =\frac{1}{r} \frac{2}{\pi} \int_{0}^{2 k_{F} r} \frac{\sin x}{x} d x
\end{aligned}
$$

Since $k_{F}$ is related to the density, it is now straightforward to generalize this to inhomogeneous systems. We simply take $r \rightarrow\left|\mathbf{r}-\mathbf{r}^{\prime}\right|$ and $k_{F} \rightarrow\left[3 \pi^{2} \tilde{n}\left(\mathbf{r}, \mathbf{r}^{\prime}\right)\right]^{1 / 3}$ with $\tilde{n}\left(\mathbf{r}, \mathbf{r}^{\prime}\right)=$ $\left[n(\mathbf{r})+n\left(\mathbf{r}^{\prime}\right)\right] / 2$. Thus, we obtain a nonlocal functional with no free parameters by performing a simple local renormalization of the correlation hole. It can be regarded as an ALDA functional where the delta function in Eq. (3) has acquired a density-dependent broadening. At large separation it reduces to the pure Coulomb interaction and it is expected to retain the accurate description of van der Waals interactions characteristic of RPA. For example, in a jellium with $r_{s}=2.0$ two points separated by $5 \AA$ give a renormalized interaction $v^{r}\left[r_{s}=2\right]\left(\left|\mathbf{r}-\mathbf{r}^{\prime}\right|\right)=0.97 v\left(\left|\mathbf{r}-\mathbf{r}^{\prime}\right|\right)$ and the magnitude of the Coulomb part of the kernel is a factor of 30 larger than $f_{x}^{\mathrm{rALDA}}$.

The renormalized ALDA functional has been implemented in the DFT code GPAW, ${ }^{26,27}$ which uses the projector augmented wave (PAW) method. ${ }^{28}$ The response function is calculated in a plane wave basis set as described in Ref. 29. The coupling constant integration is evaluated using eight Gauss-Legendre points and the frequency integration is performed with 16 Gauss-Legendre points with the highest point situated at $800 \mathrm{eV}$. Since the kernel Eq. (5) is only invariant under simultaneous lattice translation in $\mathbf{r}$ and $\mathbf{r}^{\prime}$, its plane wave representation takes the form

$$
f_{\mathbf{G G}^{\prime}}^{\mathrm{rALDA}}(\mathbf{q})=\frac{1}{V} \int_{V} d \mathbf{r} \int_{V} d \mathbf{r}^{\prime} e^{-i \mathbf{G} \cdot \mathbf{r}} \tilde{f}\left(\mathbf{q} ; \mathbf{r}, \mathbf{r}^{\prime}\right) e^{i \mathbf{G}^{\prime} \cdot \mathbf{r}^{\prime}},
$$




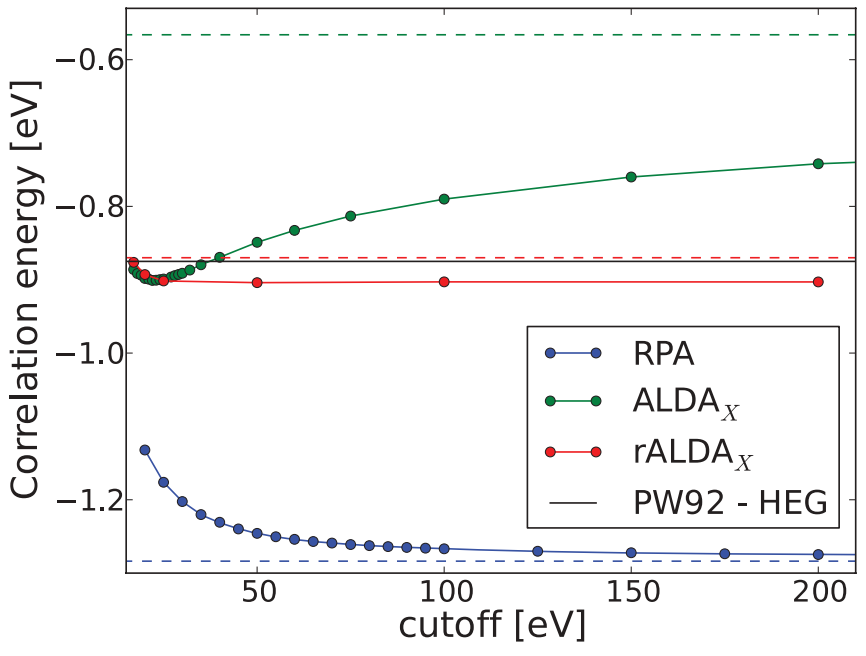

FIG. 3. (Color online) Correlation energy per valence electron in $\mathrm{Na}$ evaluated with RPA, ALDA, and rALDA. The dashed lines show the values obtained with the functionals for the homogeneous electron gas using the average valence density of $\mathrm{Na}$.

where $\mathbf{G}$ and $\mathbf{G}^{\prime}$ are reciprocal lattice vectors, $\mathbf{q}$ belongs to the first Brillouin zone, and

$$
\tilde{f}\left(\mathbf{q} ; \mathbf{r}, \mathbf{r}^{\prime}\right)=\frac{1}{N} \sum_{i, j} e^{i \mathbf{q} \cdot \mathbf{R}_{i j}} e^{-i \mathbf{q} \cdot\left(\mathbf{r}-\mathbf{r}^{\prime}\right)} f\left(\mathbf{r}, \mathbf{r}^{\prime}+\mathbf{R}_{i j}\right) .
$$

Here we have introduced the lattice point difference $\mathbf{R}_{i j}=$ $\mathbf{R}_{i}-\mathbf{R}_{j}$ and the number of sampled unit cells $N$ ( $k$ points). $\tilde{f}\left(\mathbf{q} ; \mathbf{r}, \mathbf{r}^{\prime}\right)$ is thus periodic in both $\mathbf{r}$ and $\mathbf{r}^{\prime}$ and $f_{\mathbf{G G}^{\prime}}^{\mathrm{rALDA}}(\mathbf{q})$ should be converged by sampling a sufficient number of nearest neighbor unit cells. While the response function is calculated within the full PAW framework, it is not trivial to obtain the PAW corrections for a nonlocal functional and we use the bare $\mathrm{ALDA}_{X}$ kernel to calculate contributions to the rALDA kernel from the augmentation spheres. ${ }^{29}$

As a first test of the functional for ab initio applications, we have calculated the correlation energy of the valence electrons of bulk Na. We do not have a number for the exact value of the correlation energy, but due to the delocalized nature of the valence electrons it is expected that the result should be close to the correlation energy of the homogeneous electron gas at the average valence density of $\mathrm{Na}$. This is supported by the close agreement between the RPA correlation energy of $\mathrm{Na}$ and the homogeneous electron gas. ${ }^{30} \mathrm{We}$ found the rALDA calculations to be converged when two nearest unit cells were included. The result is shown in Fig. 3 as a function of plane wave cutoff energy along with the RPA and $\mathrm{ALDA}_{X}$ results. As expected, RPA underestimates the correlation energy while $\mathrm{ALDA}_{X}$ overestimates it. Again, one should note the slow convergence of the $\mathrm{ALDA}_{X}$ calculation originating from the $q$-independent kernel. For plane wave implementations, an additional problem is posed by the divergens of $f_{x}^{\mathrm{ALDA}} \sim n^{-2 / 3}$ at small densities. A particularly nice feature of the kernel (5) is that the small density divergence of ALDA is regulated. For example, for small $r=\left|\mathbf{r}-\mathbf{r}^{\prime}\right|$ one obtains

$$
f_{x}^{\mathrm{rALDA}}[n](r)=4 n f_{x}^{\mathrm{ALDA}}[n],
$$

whereas ALDA diverges.
TABLE I. Correlation energies of $\mathrm{H}, \mathrm{H}_{2}$, and $\mathrm{He}$ evaluated with different functionals. Exact values are taken from Ref. 31. All numbers are in $\mathrm{kcal} / \mathrm{mol}$.

\begin{tabular}{lcccccc}
\hline \hline & LDA & PBE & RPA & ALDA $_{X}$ & rALDA & Exact \\
\hline $\mathrm{H}$ & -14 & -4 & -13 & 6 & -2 & 0 \\
$\mathrm{H}_{2}$ & -59 & -27 & -51 & -16 & -28 & -26 \\
$\mathrm{He}$ & -70 & -26 & -41 & -19 & -27 & -26 \\
\hline \hline
\end{tabular}

The accuracy of molecular atomization energies by RPA is comparable to that of PBE, however, total correlation energies are typically severely underestimated. ALDA, on the other hand, tends to overestimate total correlation energies by approximately the same amount. This is clearly seen for homogeneous systems displayed in Figs. 2 and 3 and the trend is also observed for inhomogeneous systems. In Table I we show a few examples of atomic and molecular correlation energies calculated with the rALDA functional and compared with LDA, PBE, RPA, and ALDA results. The ACDF correlation energies were calculated in a $6 \times 6 \times 7 \AA$ unit cell. The RPA and rALDA results were calculated at increasing cutoffs up to $400 \mathrm{eV}$ and extrapolated to infinity. The $\mathrm{ALDA}_{X}$ results were extrapolated from $1000 \mathrm{eV}$, but are still not well converged with respect to cutoff and represent a lower bound on the absolute $\mathrm{ALDA}_{X}$ correlation energies. It is clear that the rALDA functional performs much better than both RPA and ALDA.

The significantly improved total correlation energies are a very nice feature of the rALDA kernel. However, most physical properties depend on energy differences and the kernel is not of much use if it does not perform at least as well as RPA for such quantities. In Table II, we display the atomization energies of a few simple molecules calculated with different methods. The RPA@LDA and RPA@PBE columns show the sum of HartreeFock and RPA energies evaluated at self-consistent LDA and PBE orbitals, respectively. Whereas the Hartree-Fock term is nearly independent of input orbitals, the RPA correlation energies show a significant dependence on the ground state functional. This dependence is unfortunate since there is no obvious choice for the set of input orbitals. In contrast, when an adiabatic approximation for the exchange-correlation kernel is

TABLE II. Atomization energies of diatomic molecules. The ALDA values are taken from Ref. 2 and experimental values (corrected for zero point vibrational energies) are taken from Ref. 32. Results in parentheses are from Ref. 6. All number are in $\mathrm{kcal} / \mathrm{mol}$. The bottom line shows the mean absolute error for this small test set.

LDA PBE RPA@LDA RPA@PBE ALDA rALDA Expt.

\begin{tabular}{llllllll}
\hline $\mathrm{H}_{2}$ & -113 & -105 & -109 & $-109(109)$ & -110 & -107 & -109 \\
$\mathrm{~N}_{2}$ & -268 & -244 & -225 & $-224(223)$ & -229 & -226 & -228 \\
$\mathrm{O}_{2}$ & -174 & -144 & -103 & $-112(113)$ & -155 & -118 & -120 \\
$\mathrm{CO}$ & -299 & -269 & -234 & $-242(244)$ & -287 & -253 & -259 \\
$\mathrm{~F}_{2}$ & -78 & -53 & -13 & $-30(30)$ & -74 & -39 & -38 \\
$\mathrm{HF}$ & -161 & -142 & -122 & $-130(133)$ & -157 & -136 & -141 \\
$\mathrm{H}_{2} \mathrm{O}$ & -266 & -234 & -218 & $-222(223)$ & -249 & -225 & -233 \\
$\mathrm{MAE}$ & 33 & 10.1 & 14.9 & 8.4 & 19 & 3.7 & \\
\hline \hline
\end{tabular}


used, a consistent choice is the ground state functional from which the kernel was derived. ${ }^{2}$ In the present case of ALDA and rALDA we thus only consider calculations on top of the LDA ground state. For these molecules the rALDA kernel is seen to underbind by a few $\mathrm{kcal} / \mathrm{mol}\left(\mathrm{F}_{2}\right.$ excepted) but is superior to the RPA and ALDA results.

The additional computational cost of calculating the kernel is insignificant compared to evaluating the noninteracting response function and inverting the Dyson equation. For a pure exchange kernel, it is possible to perform the coupling constant integration analytically, however, it involves an inversion of the noninteracting response function, which may become near singular at particular frequencies. The numerical coupling constant integration thus represents an additional computational cost compared to RPA calculations.

In summary, we have presented a parameter-free exchange kernel for total correlation energy calculations within the ACDF formalism. The kernel largely cancels the selfcorrelation energy of RPA and seems to perform better than both RPA and ALDA for molecular atomization energies as well as for simple metals. Although more benchmarking is needed, these preliminary results indicate that the rALDA functional is clearly superior to RPA. In contrast to RPA, the functional has the very good feature that it provides a consistent choice of input orbitals beyond the Hartree approximation. Finally, it will be straightforward to extend the kernel to include ALDA correlation, which might be expected to improve results further, but we will leave this to future work. In fact, the renormalization method naturally generalizes to all semilocal adiabatic approximations, which all suffer from the same pathological behavior in their pair-distribution functions, and the present work just represents a single example of an entire class of renormalized adiabatic exchange-correlation kernels.

This work was supported by The Danish Council for Independent Research through the Sapere Aude program and the Danish Center for Scientific Computing. *tolsen@ fysik.dtu.dk

${ }^{1}$ M. Lein, E. K. U. Gross, and J. P. Perdew, Phys. Rev. B 61, 13431 (2000).

${ }^{2}$ F. Furche and T. V. Voorhis, J. Chem. Phys. 122, 164106 (2005).

${ }^{3}$ H. Eshuis, J. E. Bates, and F. Furche, Theor. Chem. Acc. 131, 1084 (2012).

${ }^{4}$ J. F. Dobson and J. Wang, Phys. Rev. B 62, 10038 (2000).

${ }^{5}$ J. F. Dobson and J. Wang, Phys. Rev. Lett. 82, 2123 (1999).

${ }^{6}$ F. Furche, Phys. Rev. B 64, 195120 (2001).

${ }^{7}$ J. Harl and G. Kresse, Phys. Rev. B 77, 045136 (2008).

${ }^{8}$ J. Harl and G. Kresse, Phys. Rev. Lett. 103, 056401 (2009).

${ }^{9}$ J. Harl, L. Schimka, and G. Kresse, Phys. Rev. B 81, 115126 (2010).

${ }^{10}$ S. Lebègue, J. Harl, T. Gould, J. G. Ángyán, G. Kresse, and J. F. Dobson, Phys. Rev. Lett. 105, 196401 (2010).

${ }^{11}$ L. Schimka, J. Harl, A. Stroppa, A. Grüneis, M. Marsman, F. Mittendorfer, and G. Kresse, Nat. Mater. 9, 741 (2010).

${ }^{12}$ T. Olsen, J. Yan, J. J. Mortensen, and K. S. Thygesen, Phys. Rev. Lett. 107, 156401 (2011).

${ }^{13}$ F. Mittendorfer, A. Garhofer, J. Redinger, J. Klimes, J. Harl, and G. Kresse, Phys. Rev. B 84, 201401 (2011).

${ }^{14}$ J. F. Dobson and T. Gould, J. Phys.: Condens. Matter 23, 073201 (2012).

${ }^{15}$ Z. Yan, J. P. Perdew, and S. Kurth, Phys. Rev. B 61, 16430 (2000).
${ }^{16}$ A. Grüneis, M. Marsman, J. Harl, L. Schimka, and G. Kresse, J. Chem. Phys. 131, 154115 (2009).

${ }^{17}$ X. Ren, A. Tkatchenko, P. Rinke, and M. Scheffler, Phys. Rev. Lett. 106, 153003 (2011).

${ }^{18}$ M. Fuchs and X. Gonze, Phys. Rev. B 65, 235109 (2002).

${ }^{19}$ J. Jung, P. Garcia-González, J. F. Dobson, and R. W. Godby, Phys. Rev. B 70, 205107 (2004).

${ }^{20}$ A. Heßelmann and A. Görling, Phys. Rev. Lett. 106, 093001 (2011).

${ }^{21}$ M. Hellgren and U. von Barth, Phys. Rev. B 78, 115107 (2008).

${ }^{22}$ J. P. Perdew and Y. Wang, Phys. Rev. B 46, 12947 (1992).

${ }^{23}$ M. Petersilka, U. J. Gossmann, and E. K. U. Gross, Phys. Rev. Lett. 76, 1212 (1996).

${ }^{24}$ M. Corradini, R. DelSole, G. Onida, and M. Palummo, Phys. Rev. B 57, 14569 (1998).

${ }^{25}$ C. F. Richardson and N. W. Ashcroft, Phys. Rev. B 50, 8170 (1994).

${ }^{26}$ J. J. Mortensen, L. B. Hansen, and K. W. Jacobsen, Phys. Rev. B 71, 035109 (2005).

${ }^{27}$ J. Enkovaara et al., J. Phys.: Condens. Matter 22, 253202 (2010).

${ }^{28}$ P. E. Blöchl, Phys. Rev. B 50, 17953 (1994).

${ }^{29}$ J. Yan, J. J. Mortensen, K. W. Jacobsen, and K. S. Thygesen, Phys. Rev. B 83, 245122 (2011).

${ }^{30}$ T. Miyake, F. Aryasetiawan, T. Kotani, M. van Schilfgaarde, M. Usuda, and K. Terakura, Phys. Rev. B 66, 245103 (2002).

${ }^{31}$ J. S. Lee and S. Y. Park, J. Chem. Phys. 112, 10746 (2000).

${ }^{32}$ A. Karton, E. Rabinovich, J. M. L. Martin, and B. Ruscic, J. Chem. Phys. 125, 144108 (2006). 